\title{
The analysis of volatile flavor components of Jin Xiang garlic and Tai'an garlic
}

\author{
Changsong Shan ${ }^{1}$, Chao Wang $^{2}$, Jin Liu ${ }^{3}$, Peng Wu ${ }^{1 \#}$ \\ ${ }^{1}$ College of Food Science and Engineering, Shandong Agricultural University, Tai' an, China; \\ ${ }^{\#}$ Corresponding Author: wupengguai@163.com (Peng Wu), hzscs518@126.com \\ ${ }^{2}$ College of Horticulture Science and Engineering, Shandong Agricultural University, Tai' an, China \\ ${ }^{3}$ School of Biological Science, Taishan Medical University, Tai'an, China
}

Received 4 November 2013; revised 4 December 2013; accepted 15 December 2013

Copyright (C) 2013 Changsong Shan et al. This is an open access article distributed under the Creative Commons Attribution License, which permits unrestricted use, distribution, and reproduction in any medium, provided the original work is properly cited.

\section{ABSTRACT}

The volatile flavor compounds of Jin Xiang garlic and Tai'an garlic in chemical composition were detected and analyzed and the contents of them were compared and determinated. The volatile constituents of Jin Xiang garlic and Tai'an garlic were compared and analyzed by automatic static headspace and gas chromatography-mass spectrometry. Qualitative analysis of samples was made through the analysis of gas chromatography-mass spectrometry and NIST mass spectral library computer retrieval, and quantitative analysis was made by using area normalization method. The analysis results show that the slight difference of the volatile flavor compounds was detected in different places of origin garlic and Jin Xiang garlic was detected more total sulfur-containing compounds than Tai'an garlic. Meanwhile, the contents of sulfur compounds of the fresh garlic were more than the stored garlic and there were significant differences between them. The tests results indicated that flavor substances' types were slightly different between Jin Xiang garlic and Tai'an garlic, and regional differences cannot affect the garlic flavor substances type. Jin Xiang garlic has more obvious flavor substances than Tai'an garlic which play a decisive role in the garlic flavor, such as 1,3-dithiane, and allyl trisulfide and allyl disulfide and diallyl tetrasulphide. The result of this research indicates that Automatic static headspace and gas chromatography-mass spectrometry is a fast, easy, efficient and accurate method to analyze and identify the volatile

"Supported by Knowledge Innovation Program of the Technology Bureau of Tai' an (No. 2011D2013). flavor components of garlic.

Keywords: Garlic; Automatic Static Headspace; Gas Chromatography-Mass Spectrometry Analysis; Flavor; Components Analysis

\section{INTRODUCTION}

Garlic is liliaceous biennial herbaceous plants underground bulb of garlic, spicy taste, strong garlic smell [1]. It is well known that garlic is abundant of garlincin, alliinase, allin, alanine [2]. Studies have shown that the main bioactive substances of garlic are the alliin and the organic compounds containing sulfur which was generated by endogenous alliinase reaction, such as thio-dipropylene, diallyl thisulfide. Those organic compounds, such as sulfur, not only have the efficacy of sterilization and antiphlogistic, reducing serum cholesterol and triglyceride, and prevention of coronary heart disease and cerebral thrombosis $[3,4]$, but also can strengthen power for the prevention of cancers [5]. Jin Xiang garlic has many good properties, such as deliciousness, pungency purity, crispy and tasty product, mildew resistance, rot-fastness, storable character and high nutritive value. Therefore Jin Xiang garlic is widely regarded as a kind of high-valuable vegetable.

In recent years, steam distillation (SD), solvent extraction, simultaneous distillation extraction (SDE) and headspace solid-phase micro-extraction (HS-SPME) were used to study volatile flavor compounds, and Mr. Lee found that HS-SPME was better than other methods in identifying the garlic flavor substances [6-9]. Compared with those technologies, Mr. Lee found that HS-SPME has a better effect on identifying garlic flavor substances [10]. Based on the fact that allicin is easy to volatilize, Mondy made a comparison between GC-MS and HPLC [11]. The results showed that the choice of analytic in- 
struments had a great influence on analysis results, especially the volatile substances. But they were not involved in the automation and static headspace GC analysis methods. Automated static headspace analysis, which has the advantage of speediness, high degree of automation, less sample consumption, low test cost and avoiding solvent residual at the greatest degree, got rapid development in the analysis of the volatile oil recently.

Studies have shown that the garlic has well performance not only on nutritional value but also the medicinal. In recent years, the studies of Jin Xiang garlic are focus on its market research, and there are few experimental studies on the volatile substances of Jin Xiang garlic. Different kinds of those volatile substances, however, might play different roles in the medical science according to the studies. Studies have shown that allyl trisulfide performs better on resisting hyperlipidemia and infection, while on the other hand, allyl disulfide does better on resist oxidation and caducity. This difference between allyl trisulfide and allyl disulfide puts forward different requirements for the quality of garlic. It seems it is hard to estimate the quality of garlic only by judging the content of allyl trisulfide and the physical index such as specific rotation, refractive index and proportion determined under the standard of FCC during the process of export.

The garlic could be classified into two kinds by the color of skin, purple skin garlic and white skin garlic. As a representative resource, Jin Xiang garlic has attracted people's attention widely owning to its excellent quality. Jin Xiang is the largest area planting garlic in China, and about 1 million tons garlic are produced each year, accounting for $70 \%$ of the garlic exports. The establishment and improvement of Jin Xiang garlic market played an important role on the country garlic market stability. Jin Xiang has put the garlic as the leading industry to promote development [12] since 2005, and this strategy provided an irreplaceable contribution on its way to become the hometown of Chinese garlic and the world's largest garlic processing and stored center. As a result, studies on the components of Jin Xiang garlic could do great favor to the quality detection both in the internal and international market.

This research took Jin Xiang garlic and Tai'an garlic for material and adopted the automatic static headspace and GC-MS method to make a comparison between them about volatile substances. Meanwhile it can provide theoretical basis for the further research on Jin Xiang and Tai'an garlic.

\section{Materials and Methods}

\subsection{Experimental Materials}

Jin Xiang purple skin garlic (cold stored, fresh), bought in Jin Xiang farmers' markets. Tai'an purple skin garlic (cold stored, fresh), from Tai'an farmers' markets.

\subsection{Main Instruments}

GC-MS QP 2010 Plus, Shimadzu Corporation. Turbo Matrix Headspace Sampler, American Platinum-El companies. Chromatographic column is Rex-5 (30 $\mathrm{m} \times 0.32$ $\mathrm{mm} \times 0.25 \mu \mathrm{m})$.

\subsection{Instrumental Analysis}

Take fresh and stored garlic from Jin Xiang and Tai'an respectively for materials, peeled and washed, cut into slices and put $3.0 \mathrm{~g}$ respectively into $20 \mathrm{~mL}$ headspace sample bottle, sealing top rapidly, keeping 30 minutes at $40^{\circ} \mathrm{C}$, and then analyzed by GC-MS.

\subsection{GC-MS Analysis of Test Condition}

Chromatographic columns used was Rex-5 column $(30 \mathrm{~m} \times 0.32 \mathrm{~mm} \times 0.25 \mu \mathrm{m})$. Helium was used as carrier gas at a flow of $2.97 \mathrm{ml} \cdot \mathrm{min}^{-1}$. Temperature programming: the column temperature was maintained at $40^{\circ} \mathrm{C}$ for $2 \mathrm{~min}$, programmed at $8^{\circ} \mathrm{C} / \mathrm{min}$ to $90^{\circ} \mathrm{C}$, then 4 ${ }^{\circ} \mathrm{C} / \mathrm{min}$ to $120^{\circ} \mathrm{C}$ and then at $10^{\circ} \mathrm{C} / \mathrm{min}$ to $200^{\circ} \mathrm{C}$, which was held for $5 \mathrm{~min}$. The mass spectrometer was operated in electron impact (EI) ionization mode with electron energy of $70 \mathrm{eV}$ and temperature $230^{\circ} \mathrm{C}$. The transfer line temperature was $200^{\circ} \mathrm{C}$. Scan range was $45-450 \mathrm{~m} / \mathrm{z}$.

\subsection{Qualitative and Quantitative Methods}

Methods of qualitative analysis: According to the GCMS information obtained by the computer using a standard NIST08 gallery retrieve, compared with standard spectra, and type of components of volatile compounds are obtained.

Quantitative analysis method: peak area normalization method for their relative content.

\section{Results}

\subsection{GC-MS Total Ion Chromatograms of Garlic Samples}

Gas chromatography data processing system, the computer retrieval and NIST library (107 k compounds) and Wiley library (y320 k compounds, version 6.0), combined with the artificial map matching were used to confirm all kinds of chemical composition of volatile substances. According to peak area normalization method for their relative content, only when the matching degree and purity is greater than 80 (maximum 100) identification results will be reported.

Different kinds of samples were analyzed by GC-MS to get the total ion chromatograms, by using qualitative and quantitative analysis methods, and with the aid of 
computer retrieval and NIST library, calculating the result by peak area normalization method.

According to the Figure 1, the volatile components types of four garlic samples detected were slightly different from each other. The kinds of substances of stored purple garlic (Samples 1 and 2) were more than fresh purple Garlic (Samples 3 and 4). The retention time of the major sulfur compounds, such as 1,3-two thiophene, allyl disulfide, allyl trisulfide and diallyl tetrasulphide was the same retention time, but the content were different.

\subsection{Results and Analysis of Volatile Flavor Substances of Samples}

Volatiles of different garlic samples were evaluated with the method of HS-SPME-GC-MS and search into computer library. There are twenty-five kinds of volatiles were detected in different individuals (Table 1). There are 20 and 19 volatiles in stored and fresh Jin Xiang garlic and 13 and 14 volatiles in stored and fresh Tai'an garlic.

As can be seen in Table 1, The main volatile flavor substances in garlic is 1,3-two thiophene, allyl disulfide, allyl trisulfide and diallyl tetrasulphide. Among them, the relative content of most is allyl trisulfide (allicin), according for the total volatiles of $25.22 \%, 22.46 \%$, $39.97 \%$ and $38.01 \%$ in stored Jin Xiang and Tai'an garlic and fresh Jin Xiang and Tai'an garlic respectively. Followed by are allyl disulfide and diallyl tetrasulphide.

Experimental results show that there is more allyl trisulfide and allyl disulfide in the fresh garlic than that in the stored garlic, but the content of diallyl tetrasulphide in the fresh garlic is significantly lower than that in the stored garlic. Meanwhile, regional differences have slight influence on garlic volatile flavor components, but tremendous effects on the relative percentage content.

\section{Conclusions and Discussion}

\subsection{Components of Garlic Volatile Flavor}

The volatile constituents of Jin Xiang and Tai'an garlic were detected by automatic static headspace and gas chromatography-mass spectrometry. Qualitative analysis of samples was made through the analysis of NIST mass spectral library computer retrieval, and quantitative analysis was made by area normalization method. Results showed that 1,3-Dithiane, and Allyl trisulfide and Allyl disulfide and Diallyl tetrasulphide were the major volatile flavor of garlic. And the content of allyl trisulfide (allicin) is relatively higher than others. Because of different producing area and stored time, the composition of garlic volatile flavor is slightly different and regional difference is not obvious. All this provided a scientific basis on the appraisal of the quality of garlic. Further-

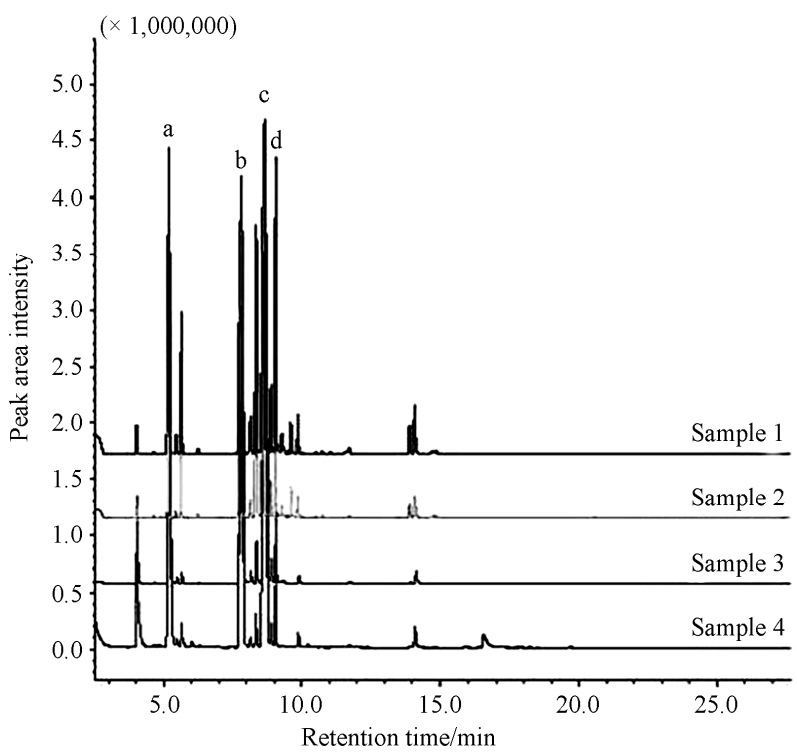

"1" for the stored of Jin Xiang purple garlic Atlas of sample ions; "a" for the 1,3-two thiophene; " 2 " for the stored of Tai' an purple garlic Atlas of sample ions; "b" for the allyl disulfide; " 3 " for Jin Xiang fresh purple skin garlic sample ion spectrum; "c" for the allyl trisulfide; "4" for Tai'an fresh purple skin garlic sample ion spectrum; "d" for the diallyl tetrasulphide.

Figure 1. Total ion chromatogram of volatile compounds of four kinds of garlic.

more, experiment results showed that there were 19 and 20 compounds in stored Tai'an and Jin Xiang garlic, but only 14 and 13 compounds in fresh Tai'an and Jin Xiang garlic. The difference of producing area and stored time might contribute much to this. Compared with the literature reported, 14 compounds are consistent [13-15] with them, with almost all the major volatile flavor included.

Unfortunately, the other kinds of components, such as alliin and allitol, were not detected in this experiment. The reason might be that the alliin and allitol existed in garlic phosphorus buds in the form of stable and odorless $[16,17]$, When facing with the external force, the garlic neuraminidase, from different organization with alliin, would contact with alliin. Enzymatic bio-chemical reaction will occur under the catalysis of alliin enzyme, and generate allicin, with strong stimulating smell (Double alkene propyl glucosinolates sulfinic acid ester) [18].

\subsection{The Content of Volatile Flavor Compounds in Garlic}

In this study, the major substances, which play a decisive role in the garlic flavor, were 1,3-Dithiane, Allyl trisulfide, Allyl disulfide and Diallyl tetrasulphide. From Table 1, we know that the content of allyl trisulfide is higher than that in the garlic in the literature reports of Zhou Jianglu and Wang Ying [13,14]. Therefore, the content of allyl trisulfide (allicin) and allyl disulfide were different from each producing areas. This may be related to the moisture content and varieties of garlic in different 
Table 1. Volatiles in four kinds of garlic samples.

\begin{tabular}{|c|c|c|c|c|c|c|c|}
\hline \multirow[b]{2}{*}{ Number } & \multirow[b]{2}{*}{$\begin{array}{l}\text { Retention } \\
\text { time/min }\end{array}$} & \multirow[b]{2}{*}{$\begin{array}{l}\text { Matching } \\
\text { degree } / \%\end{array}$} & \multirow[t]{2}{*}{ Name } & \multicolumn{4}{|c|}{ Relative percentage content $/ \%$} \\
\hline & & & & $\begin{array}{l}\text { Jin Xiang } \\
\text { stored purple } \\
\text { skin }\end{array}$ & $\begin{array}{l}\text { Tai'an stored } \\
\text { purple skin }\end{array}$ & $\begin{array}{l}\text { Jin Xiang } \\
\text { fresh purple } \\
\text { skin garlic }\end{array}$ & $\begin{array}{l}\text { Tai'an fresh } \\
\text { purple skin } \\
\text { garlic }\end{array}$ \\
\hline 1) & 4.019 & 94 & Allyl sulfide & 0.91 & 0.52 & 3.87 & 5.07 \\
\hline 2) & 4.650 & 80 & 1-Propene, 1,1'-thiobis- & 0.07 & 0.09 & - & - \\
\hline 3) & 4.956 & 92 & 3,4-Dimethylthiophene & - & - & - & - \\
\hline 4) & 5.214 & 85 & 1,3-Dithiane & 16.17 & 9.51 & 17.21 & 24.27 \\
\hline 5) & 5.451 & 80 & Disulfide, methyl 1-propenyl & 0.75 & 0.36 & 0.30 & 0.22 \\
\hline 6) & 5.647 & 83 & Disulfide, methyl 2-propenyl & 4.21 & 3.53 & 0.44 & 0.75 \\
\hline 7) & 6.012 & 81 & Ethanethioamide, N, N-dimethyl- & - & - & - & 0.23 \\
\hline 8) & 6.242 & 96 & Dimethyl trisulfide & 0.17 & 0.12 & - & - \\
\hline 9) & 7.794 & 86 & Allyl disulfide & 20.06 & 19.61 & 30.26 & 25.65 \\
\hline 10) & 8.143 & 83 & 2-Vinyl-1,3-dithiane & 1.36 & 1.25 & 1.98 & 0.96 \\
\hline 11) & 8.355 & 84 & 3-(Allylsulfanyl) propanoic acid & 9.49 & 15.63 & 1.98 & 0.96 \\
\hline 12) & 8.884 & 83 & 2-Ethylidene [1,3] dithiane & 2.20 & 2.15 & - & - \\
\hline 13) & 8.705 & 86 & Allyl trisulfide & 25.22 & 22.46 & 39.97 & 38.01 \\
\hline 14) & 9.056 & 85 & Diallyl tetrasulphide & 12.56 & 18.56 & 3.10 & 1.79 \\
\hline 15) & 9.287 & 80 & Dioxane & 0.78 & 0.86 & 0.13 & 0.05 \\
\hline 16) & 9.358 & 83 & 3-Aminorhodanine & 0.16 & 0.15 & - & - \\
\hline 17) & 9.618 & 82 & Methyl (allylsulfanyl) acetate & 0.83 & 1.45 & - & - \\
\hline 18) & 9.875 & 86 & 6-(Methylthio)hexa-1,5-dien-3-ol & 1.19 & 1.03 & 0.33 & 0.38 \\
\hline 19) & 10.244 & 85 & 1,3,5-Trithiane & - & - & - & 0.07 \\
\hline 20) & 10.745 & 82 & 2-Mercapto-3,4-dimethyl-2,3-dihydrothiophene & 0.07 & 0.11 & - & - \\
\hline 21) & 11.721 & 94 & 3-Vinyl-3,4-dihydro-1,2-dithiine & 0.17 & - & - & - \\
\hline 22) & 13.912 & 83 & 1-Ethyl-2-methyl-4-pentenyl methyl ether & - & 0.92 & 0.61 & - \\
\hline 23) & 14.097 & 80 & 1-Heptene, 5-methoxy-4-methyl- & 1.86 & 1.50 & 0.08 & - \\
\hline 24) & 14.731 & 87 & 2,5-Dimethyl-1,3,4-thiadiazole & 0.17 & - & - & - \\
\hline 25) & 16.547 & 84 & Benzothiofuran & - & - & - & 0.86 \\
\hline
\end{tabular}

Note: “-” Not detected.

regions, and connected with the local climate, temperature and precipitation and so on. This also laid a foundation for the further study on how these factors affect the kinds of flavor substances and content.

\subsection{The Development of Garlic Volatiles in Fragrance and Flavor Industry}

Hall, R.L. and Oser, B.L. had some studies in sulfur compounds in garlic. Research showed that allyl disulfide has strong meat aroma and the taste of onions, garlic and mustard. So it can be used for product flavor of condiment and meat products [19]. Methyl-2-propylene di- sulfide also has the fragrant of onion, leek, garlic and the pickled garlic. As the allyl disulfide, it also can be used for product flavor of condiment and meat products [20]. This study shows that the contents of diallyl disulfide and diallyl trisulfide are higher both in Jin Xiang and Tai'an garlic. Therefore, this garlic also has a good direction for development in the field of flavors.

\subsection{Choice of Testing Instrument of Garlic Volatiles}

Studies have shown that the allyl sulfur compounds in garlic are not stable, especially allyl trisulfide, which is 
easy to break down at high temperature, and lead to the decrease of the allyl trisulfide (allicin) levels and the increase of other decomposition products. As a result, it also puts forward higher requirements to analytical instrument. Sun-Neo Lee and N. Mondy make a comparison to the results of garlic flavor substances through the analysis detection methods such as steam distillation (SD) and high performance liquid chromatography (HPLC) and so on, but not automation and static headspace GC analysis method. This research indicates that GC-MS is a fast, convenient, efficient and accurate method to analyze and identify the volatile flavor components of garlic flavor components types and contents.

\section{REFERENCES}

[1] Nanjing University of Chinese Medicine (1996) Chinese material medical dictionary. The Science \& Technology Press, Shanghai.

[2] Yin, H. and Zhang, J.H. (1996) Chemical constituents and pharmacological and application of garlic. Zhejiang Journal of Traditional Chinese Medicine Hospital, 20, 37-38.

[3] Yu, X.R. and Cong, Y.Z. (1994) Research advance on chemical component and pharmacological action of garlic. Chinese Herbal Medicine, 25, 158.

[4] Saied, H.D., et al. (2010) The effects of fresh garlic on the serum concentration of total cholesterol, total triglyceride and adipose tissues of broilers. Comparative Clinical Pathology, 19, 363-365. http://dx.doi.org/10.1007/s00580-009-0879-7

[5] Bruno, C., et al. (1998) Garlic,onion and cereal fibre as protective factors for breast cancer: A French case-control study. European Journal of Epidemiology, 14, 737-747. http://dx.doi.org/10.1023/A:1007512825851

[6] Liu, L.M., Sun, Z.D., Wang, R.H., et al. (2007) Extraction process of steam distillation of volatile oil of garlic. Chinese Journal of Traditional Chinese Medicine, 32, 744-746.

[7] Yang, P., Yang, G.M., Zhang, X., et al. (2007) GC-MS analysis of plumula nelumbinis of lipid-soluble components from different producing area. Journal of Nanjing University of Traditional Chinese Medicine, 23, 374-377.
[8] Ren, S.H., Guo, Y., et al. (2004) GC-MS analysis of volatile components of Wumei from different producing area. Chinese Herbal Medicines, 27, 16-19.

[9] Pu, L.X., Tang, T.J., Yuan, X.H., et al. (2010) Analysis on volatile constituents of aralia cordata thumb from different places. Journal of Anhui Agricultural Sciences, $\mathbf{3 8}$, 8946-8948.

[10] Lee, S.-N., et al. (2003) Comparative study of extraction techniques for determination of garlic flavor components by gas chromatography-Mass spectrometry. Analytical and Bioanalytical Chemistry, 377, 749-756. http://dx.doi.org/10.1007/s00216-003-2163-z

[11] Mondy, N., et al. (2001) Comparison of GC-MS and HPLC for the analysis of Allium Volatiles. 53, S-356-S-360.

[12] Ma, W.-X. and Yang, Z.-L. (2001) The biological characteristics and utilization value of Jin Xiang garlic. Journal of Special Economic Animals and Plants, 5, 28-29.

[13] Zhou, J.J. (2010) HP-SPME-GC-MS analysis of volatile flavor components of garlic. Chinese Condiment, 35, 95-98.

[14] Wang, Y., Wu, W.L., Wang, B., et al. (2012) Component analysis of volatile oil of garlic from different districts and assay of garlicin content. Journal of Anhui Agricultural Sciences, 40, 5321-5323.

[15] Wang, X.F., Dong, Y. and Liu, H.L. (2008) Determination of chemical constituents of essential oil of garlic by gas chromatography mass spectrometry. Lishizhen Med Mat Med Res, 19, 71-72.

[16] Mutsch, E., et al. (1993) A novel amino acid glycoside and three amino acids from Allium Sativum. Journal of Natural Products, 56, 864-869. http://dx.doi.org/10.1021/np50096a009

[17] Kwbec, et al. (1999) Gas chromatographic determination of Salk(el1)yl-L cymene sulfoxides. Journal of Chromatography, 826, 85-94.

[18] Wu, C.M. and Chen, S.Y. (2000) Alliin enzymes and odorless garlic. Precision Medical Journal,13, 268

[19] Hall, O. (1972) Recent progress in the consideration of flavoring ingredients under the food additives amendment. 5. GRAS substances. Food Technology, 26, 36-41.

[20] Oser, H. (1978) Recent progress in the consideration of flavoring ingredients under the food additives amendment. 5. GRAS substances. Food Technology, 32, 60-70. 